\title{
Simulation des activités de dragage et de leur impact sédimentaire à l'aide d'une modélisation morphodynamique de l'embouchure de la Seine
}

\author{
B. Waeles ${ }^{*}$ et P. Le Hir \\ *IFREMER, laboratoire Dyneco-Physed, B. P. 70, 29280 Plouzané
}

\section{Résumé :}

Les activités de dragage dans l'estuaire de la Seine participent de manière significative aux transits sédimentaires. Les évolutions morphodynamiques mesurées montrent notamment un signal fort lié aux dépôts de dragage sur le site du Kannick (e.g. Delsinne, 2005). Dans le but d'une meilleure compréhension des processus liés aux activités de dragage, ces dernières sont intégrées dans le modèle morphodynamique SiAM-3D qui simule les évolutions morphologiques de l'embouchure de la Seine. Le code SiAM-3D simule les transports de sable et de vase ainsi que les processus de consolidation, le sédiment étant discrétisé en couches fines. Le modèle reproduit des volumes de sédiment à draguer très proches de ceux qu'ils sont en réalité (environ 3.5 millions de $\mathrm{m}^{3} / \mathrm{an}$ ) et la nature simulée des sédiments dragués $(\sim 70 \%$ de sable) est réaliste. L'effet différentiel à court terme des évolutions morphodynamiques à l'embouchure, lié aux rejets de dragage, est également décrit. Le modèle pourrait être utilisé pour optimiser les stratégies de dragage.

\section{Abstract :}

The dredging activities within the Seine significantly contribute to the sediment fluxes. The measured Morphodynamic evolutions show a strong signature related to the dumped sediments on the dedicated Kannick site (e.g. Delsinne, 2005). In order to improve our understanding concerning processes linked to the dredging activities, those are implemented in the morphodynamic model SiAM-3D which simulates the morphological evolutions of the Seine estuary. The SiAM-3D code simulates the transports of sand and mud and also the consolidation processes, the sediment being discretized in thin layers. Results show good agreement with data: first concerning the sediment volumes to be dredged (about 3.5 millions of $\mathrm{m}^{3} /$ year) and also concerning the nature of the dredged sediments ( $\sim 70 \%$ of sand). The short-term differential effect of the morphodynamic evolutions, linked to the sediment dumping, is also described. The model could be used as a tool to optimize the dredging strategies.

Mots clés: modélisation numérique, morphodynamique, dragage, clapage, sable, vase

\section{Introduction}

Les évolutions morphologiques d'environnements côtiers peuvent être significativement contraintes par des aménagements de types endiguement ou poldérisation, ou encore la mise en place et l'entretien de chenaux de navigation. C'est le cas à l'embouchure de la Seine, où la progradation des bancs à l'embouchure serait favorisée par la présence de digues submersibles qui encadrent le chenal d'accès au port de Rouen (Fig. 1). Le maintien de ce dernier nécessite d'importantes activités de dragage : environ 3,5 millions de $m^{3}$ par an (fig. 2 et Sogreah, 1997) de matériel essentiellement sableux (à 70 \%, selon Sergent et Zhang, 2001). Il a été montré que les sédiments clapés plus en aval, au niveau du « Kannick », participent DOI: 10.5150/jngcgc.2006.046-W (disponible en ligne - http://www.paralia.fr - available online) 
aux évolutions morphologiques à l'embouchure de la Seine (Delsinne, 2005); une partie des sables clapés au Kannick contribue à la progradation du banc d'Amfard situé au Nord de la digue submersible Nord.

Le devenir des sédiments clapés au Kannick a déjà fait l'objet de simulations numériques par Sergent et Zhang (2001), qui font l'hypothèse que les dépôts sur le fond présentent une forme conique et que, hormis ces dépôts, l'ensemble du fond de l'estuaire est considéré comme inérodable. La pertinence des simulations d'étalement a été validée par comparaison avec les résultats de marquages radioactifs in-situ.

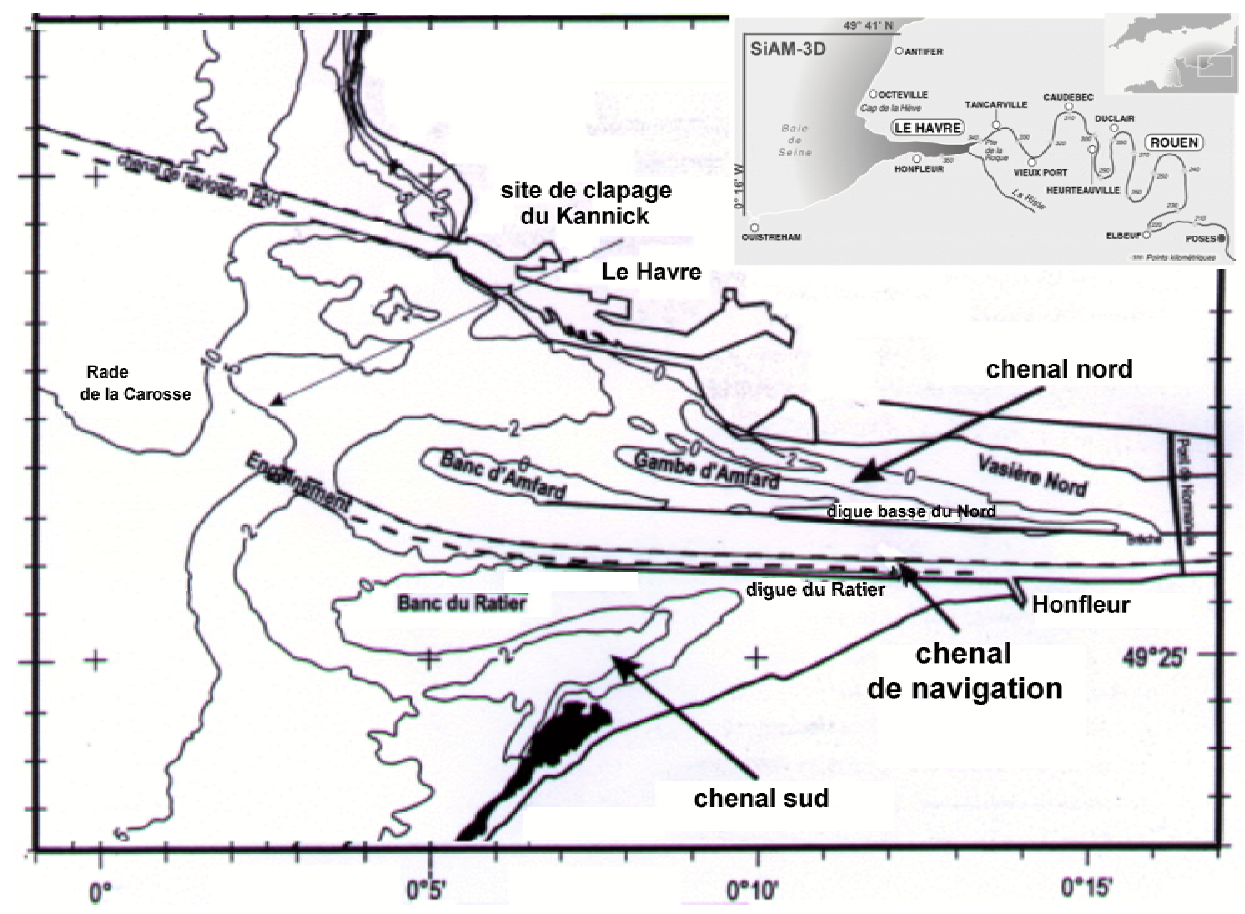

Figure 1 Détail et toponymie de la zone de l'embouchure de la Seine (bathymétrie carte SHOM de 1996), d'après Lesourd (2000). Encart : localisation de l'estuaire de la Seine et emprise et emprise du modèle SiAM-3D.

Pour la présente étude, les activités de dragage et les clapages associés sont considérés de manière globale, c'est à dire comme faisant partie des transports sédimentaires qui ont lieu au sein du système de l'estuaire de Seine. Dans le but d'une meilleure compréhension des processus sédimentaires liés aux activités de dragage, ces dernières sont prises en compte de manière schématique dans un modèle numérique morphodynamique de l'embouchure de la Seine, le modèle SiAM-3D. Les caractéristiques principales du modèle morphodynamique sont d'abord rappelées, puis la méthode pour simuler des activités de dragage est présentée. Des évolutions morphodynamiques pour un forçage réaliste (marée, débit de la Seine, vents et vagues) sont calculées sur plusieurs années. Les résultats décrits portent sur les volumes et la nature des sédiments dragués dans le chenal d'accès au Port de Rouen, ainsi que sur l'impact sédimentologique des dépôts dans la zone du Kannick et son voisinage.

\section{$\underline{\text { 2. Modèle morphodynamique de l'embouchure de la Seine. }}$}

\subsection{Un modèle morphodynamique 3D}

Le modèle qui simule les évolutions morphologiques à l'embouchure de la Seine est le modèle morphodynamique SiAM-3D (Waeles et Le Hir, 2004 ; Waeles, 2005 ; Waeles et al., 
in press). Ses principales caractéristiques sont d'une part de simuler conjointement le transport des sédiments de type cohésif (sableux) et non-cohésif (vaseux), ce qui est essentiel compte tenu du fait que sable et vase contribuent aux évolutions morphodynamiques à l'embouchure de la Seine, et d'autre part de simuler la consolidation des couches de vase ou de vases sableuses, le sédiment étant discrétisé en couches fines (Waeles et al., 2005).

La limite marine Ouest du modèle se situe au méridien de Ouistreham, la limite Nord à la latitude du cap de la Hève. La limite amont du modèle, limite de l'estuaire au sens de la marée dynamique, se situe au barrage de Poses (e.g. Waeles, 2005). Le maillage horizontal du domaine est un maillage orthogonal irrégulier, les dimensions des mailles étant minimales au niveau de l'embouchure, avec une longueur de $300 \mathrm{~m}$ dans la direction Ouest-Est et une largeur de $100 \mathrm{~m}$ dans la direction Sud-Nord. Les forçages hydrodynamiques pris en compte sont la marée réelle, le débit journalier de la Seine, les vents réels mesurés au cap de la Hève et les vagues associées (reconstituées par calcul).

\subsection{Modèle hydrodynamique}

Le modèle courantologique est le code SiAM-3D (Cugier et Le Hir, 2002), caractérisé par une séparation entre les modes internes et externes: les variations de l'élévation de la surface libre sont résolues par un modèle $2 \mathrm{DH}$ pour réduire le temps de calcul. Une version du code en coordonnées réduites $\sigma$ (Waeles, 2005) est utilisée ici. La fermeture turbulente est basée sur un modèle en longueur de mélange qui tient compte de l'amortissement des turbulences par les gradients de densité. Le recouvrement/découvrement des zones intertidales est simulé ; il est tenu compte implicitement d'une variation linéaire de la surface immergée d'une maille en cours de recouvrement (découvrement) en fonction du niveau d'eau (Waeles, 2005), d'après Balzano (1998).

Les hauteurs de vagues et les contraintes sur le fond qui en résultent sont calculées par le modèle Hiswa, à partir des conditions de vagues aux limites marines liées empiriquement aux conditions de vent (intensité et direction) et aux caractéristiques du fetch dans la Baie de Seine, les houles du large ayant peu d'effet à l'embouchure de la Seine (Silva Jacinto, 2001). Des simulations préliminaires ont été réalisées sur la bathymétrie initiale pour fournir une base de données de vitesses orbitales pour différentes configurations de vagues et de hauteur d'eau (Le Hir et al., 2001). Pendant les simulations morphodynamiques, la distribution des vitesses orbitales est déduite de la base de donnée constituée, en interpolant la hauteur de vague et le niveau d'eau. Pour tenir compte des réactualisations bathymétriques, ces vitesses orbitales sont modifiées en tenant compte des variations de hauteur d'eau, pour un niveau d'eau donné, dues aux évolutions morphodynamiques (Waeles, 2005).

\subsection{Modèle de transport sédimentaire sable/vase.}

\subsection{1 transport en suspension}

Le transport de vase est simulé en suspension. C'est également le cas pour le sable ; cette approche est possible car le sable présent à l'embouchure de la Seine est un sable plutôt fin $\left(D_{50}=200 \mu \mathrm{m}\right)$. Des expressions spécifiques sont utilisées pour les flux de dépôt et d'érosion. Ainsi, un formalisme mathématique identique est utilisé pour les deux types de particules et permet une représentation multicouches du sédiment. On suppose que les particules de vase et les grains de sable sont transportés indépendamment dans la colonne d'eau. Une équation d'advection-dispersion est résolue séparément pour chaque fraction. La vitesse de chute des 
grains de sable est supposée constante, tandis que pour la fraction vaseuse elle peut varier en raison des processus de floculation et d'entravement (Le Hir et al., 2001).

La simulation du transport des particules de vase est pleinement tridimensionnelle (avec une coordonnée verticale réduite $\sigma$ ). Pour les grains de sable, on suppose que le transport a lieu uniquement dans la couche de fond (Waeles, 2005). Pour des simulations morphodynamiques à moyen terme ( plusieurs années, comme c'est le cas pour la présente étude), la résolution d'une équation d'advection-dispersion 3D serait trop coûteuse en temps calcul en raison des vitesses de chute relativement élevées des grains de sable qui nécessitent un pas de temps très petit pour assurer la stabilité du modèle 3D. Pour réduire la sous-estimation du transport de sable quand le mélange vertical turbulent est important, le flux horizontal est corrigé pour tenir compte du transport des grains de sable dans les autres couches. Pour calculer cette correction, la concentration de sable est supposée suivre un profil de Rouse (à l'équilibre); le profil étant modifié dans la couche limite de vague pour tenir compte du mélange turbulent par les vagues.

\subsection{2 flux de dépôt et d'érosion}

Les flux de dépôt de sable et de vase sont supposés indépendants. Le dépôt de sable est extrapolé à partir de la concentration dans la couche de fond en supposant localement un profil de Rouse (modifié dans la couche limite de vague). Cette procédure n'est pas appliquée pour la vase dont la faible vitesse de chute n'entraîne pas d'importants gradients verticaux de concentration.

La formulation des flux d'érosion de sédiments présents dans la couche superficielle de sédiment dépend des proportions respectives de sable et de vase. D'après Van Ledden (2001), qui s'est basé sur des expériences réalisées en canal (e.g. Panagiotopoulos et al., 1997), deux modes d'érosion sont distingués. Pour une fraction massique de vase inférieure à environ 30 $\%$, le sédiment est considéré comme non-cohésif et une loi d'érosion spécifique au sable (Waeles, 2005) est appliquée à l'ensemble du sédiment. Pour des fractions de vase supérieures, l'ensemble du sédiment est considéré comme cohésif et la loi classique de Partheniades est appliquée. Pour les deux régimes, les flux d'érosion de sable et de vase sont modulés par leurs proportions respectives dans la couche superficielle de sédiment.

De même l'érodabilité du sédiment (contrainte critique pour l'érosion) dépend des fractions respectives de sable et de vase du sédiment superficiel. L'érodabilité d'un sédiment de type cohésif (fraction de vase supérieure à $30 \%$ ) dépend aussi de son état de consolidation (Waeles, 2005) caractérisé par sa concentration relative en vase (i.e. la concentration en vase dans le volume non occupé par les grains de sable) conformément aux expériences de Migniot (1989).

\subsubsection{Constitution des couches de sédiment et consolidation des couches vaseuses}

Lors des phases de dépôt, la constitution des couches de sédiment dépend à la fois des sédiments qui se déposent mais également de la nature (cohésive ou non) de la couche de sédiment superficiel, de telle sorte qu'une sédimentation différentielle schématique est implicitement simulée (Waeles, 2005; Waeles et al., in press). Lorsque le sédiment superficiel est sableux : une nouvelle couche est créée si le sédiment qui se dépose est plutôt vaseux de manière à décrire au mieux la variabilité verticale du sédiment, tandis qu'un dépôt de sable entraîne un épaississement de la couche superficielle. Lorsque le sédiment superficiel est vaseux, on tient compte également de son état de consolidation. On suppose qu'un 
sédiment qui se dépose, qu'il soit sableux ou vaseux, ne se mélange pas à une couche superficielle consolidée. Si la couche superficielle est peu consolidée, on la mélange avec le sédiment qui se dépose pour minimiser le nombre de couches (ce mélange peut traduire aussi la sédimentation d'un dépôt sableux dans une vase peu consolidée).

La consolidation des couches vaseuses de sédiment est simulée par un modèle de type empirique (Wales, 2005). Pour chaque couche (de type vaseuse) de sédiment (pour une maille donnée), une équation différentielle temporelle est résolue pour la concentration relative de vase. La concentration relative de vase est relaxée vers une concentration maximale qui dépend du poids du sédiment (qui se trouve au-dessus) de la couche considérée. La concentration relative de vase augmente d'autant plus vite qu'elle est éloignée de cette concentration maximale. La vitesse de consolidation tient également compte explicitement de la valeur de la concentration relative, pour traduire le ralentissement du processus lors des différents stades de la consolidation.

\subsection{Couplage morphodynamique du modèle}

Le couplage morphodynamique consiste à modifier la cote du fond de chaque maille en considérant directement le bilan net (dépôt-érosion) au cours de chaque pas de temps du modèle de transport sédimentaire. La procédure est peu coûteuse et réduit les artefacts numériques (Hervouet et Villaret, 2004).

L'équation de base qui décrit cette réactualisation bathymétrique est la suivante :

$$
\frac{\partial(1-p) h_{\text {sed }}}{\partial t}=D-E
$$

p est la porosité du sédiment superficiel; dans le cas d'un fond sableux, la porosité dépend de l'arrangement des grains (Waeles, 2005).

E, D : flux d'érosion et de dépôt de sable et de vase

Le maillage du domaine (grille de calcul de type "Arakawa C") est tel que les érosions/dépôts (et donc les hauteurs de sédiment) sont calculés aux centres des mailles, tandis que les cotes du fond sont définies sur les 4 faces limitant les mailles (ces cotes représentent des seuils pour l'écoulement dans les directions $\mathrm{x}$ et $\mathrm{y}$ respectivement).

Lors de l'initialisation avec un sédiment d'épaisseur connue, les cotes d'un fond de référence, considéré comme inérodable, sont calculées. Au cours de la simulation, chaque cote du fond est alors réactualisée en ajoutant à la cote de référence l'épaisseur locale du sédiment, évaluée comme la demi somme des épaisseurs de sédiment dans les mailles situées de part et d'autre. Cette procédure est susceptible de générer une diffusion.

\subsection{Principaux résultats de la modélisation numérique sans dragages (Waeles, 2005)}

Partant d'une condition initiale arbitraire où le sédiment n'est constitué que de sable (à l'exception d'un stock de vase facilement érodable initialisé en amont de Tancarville), le modèle morphodynamique SiAM-3D s'avère capable de reproduire une distribution réaliste de la couverture superficielle de sédiment; en particulier la vasière Nord commence à se constituer dès les premiers jours simulés. La variabilité saisonnière de la couverture superficielle semble également être reproduite correctement par le modèle, l'extension des zones vaseuses étant maximale en période de crue. 
Concernant les évolutions morphodynamiques de l'embouchure, les principales tendances sont qualitativement reproduites : par exemple la progradation des bancs à l'embouchure, associée à leur rotation, de telle sorte que les extrémités Ouest des bancs s'écartent du chenal central. Les évolutions morphodynamiques simulées après un an semblent peu dépendre des régimes de forçage, malgré une grande variabilité évènementielle (Waeles, 2005). Ce résultat inattendu reste à confirmer en poursuivant les tests de sensibilité.

Les contributions respectives des sables et vases vis à vis des évolutions morphodynamiques à l'embouchure ont également été évaluées à l'aide du modèle. Une tendance forte est que l'évolution du banc du Ratier au sud résulte uniquement de la dynamique du transport de sable, tandis que l'évolution du banc d'Amfard au Nord est gouvernée à la fois par des dépôts sableux et vaseux, le banc d'Amfard correspondant à la position du maximum de turbidité à basse mer (par débit élevé).

\section{$\underline{\text { 3. Prise en compte des activités de dragage/clapage dans le modèle morphodynamique }}$ $\underline{\mathrm{SiAM} / 3 \mathrm{D}}$}

\subsection{Dragage dans le chenal de navigation}

La démarche adoptée consiste à simuler dans le modèle l'activité de dragage au gré des besoins pour maintenir la cote nominale du chenal de navigation. Ce dernier doit préalablement être défini, en sélectionnant les mailles concernées (figures 2 et 3). L'exercice est délicat, dans la mesure où le chenal réel est étroit $(200 \mathrm{~m})$ par rapport aux dimensions des mailles (au mieux $300 \times 100 \mathrm{~m}$ dans le secteur). Une contrainte est liée à la schématisation des mailles : les hauteurs de sédiment et les cotes du fond sont définies respectivement sur les bords et au centre des mailles. La solution choisie consiste à définir une côte du fond (fictive, c'est à dire qui n'est pas considérée pour le calcul de l'écoulement), dite "de référence", au centre de chaque maille du chenal : $h c$ est la moyenne des cotes des 4 bords $h x$ et hy qui caractérisent la maille $(i, j)$. Dans le cas où l'une de ces cotes représente une cote à terre ou une cote le long d'une des digues submersibles, elle n'est pas considérée dans le calcul de $h c$ Pendant le calcul morphodynamique, dès lors que la cote du fond (fictive) au centre de la maille dépasse la cote nominale de plus de $1 \mathrm{~cm}$, la cote du fond est ramenée à sa valeur nominale, et la différence, qui correspond à tout ou partie d'une ou plusieurs couches de sédiment, est soustraite de la hauteur totale de sédiment .

La bathymétrie initiale du modèle a été réalisée par le Port Autonome de Rouen en 1999 (donc avant le début des travaux d'aménagement de Port 2000). Elle a été rectifiée au niveau du chenal de navigation pour que la cote du fond n'y dépasse pas la valeur $-5 \mathrm{~m} \mathrm{CMH} \mathrm{(par}$ rapport au zéro hydrographique du Havre).

\subsection{Schématisation du clapage des sédiments sur le site du Kannick}

Pour tenir compte de la remise à disposition au système des matériaux dragués, on schématise le clapage des matériaux. Etant donné les discrétisations horizontales et verticales du domaine il n'est pas possible de reproduire la dynamique liée à la chute du " nuage » qui se forme lors du clapage de l'ensemble des sédiments, qui nécessite un maillage beaucoup plus fin (e.g. Silva Jacinto et Burel, 2003) . 


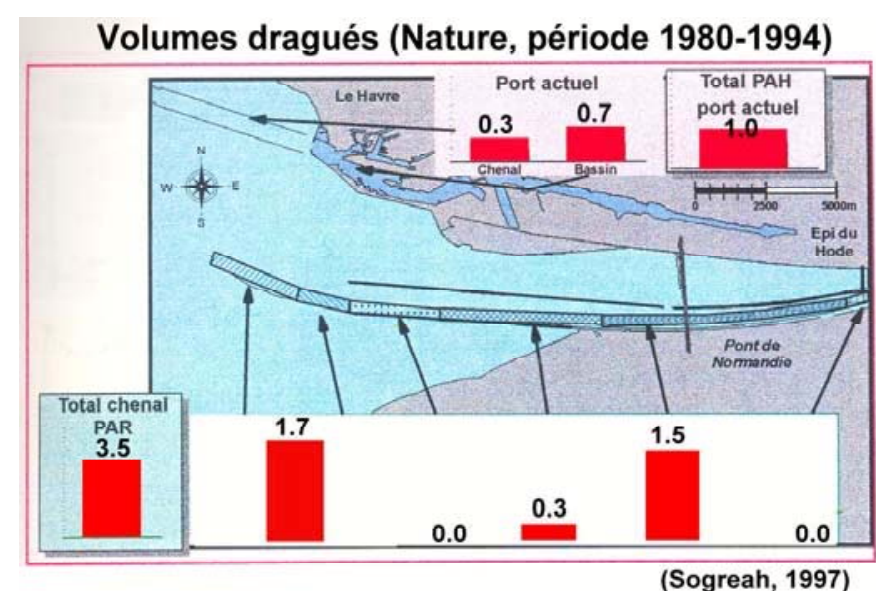

Figure 2: Répartition des volumes dragués (en millions de $\mathrm{m}^{3} / \mathrm{an}$ ) à l'embouchure de la Seine pour la période 1980-1994 (Sogreah, 1997)

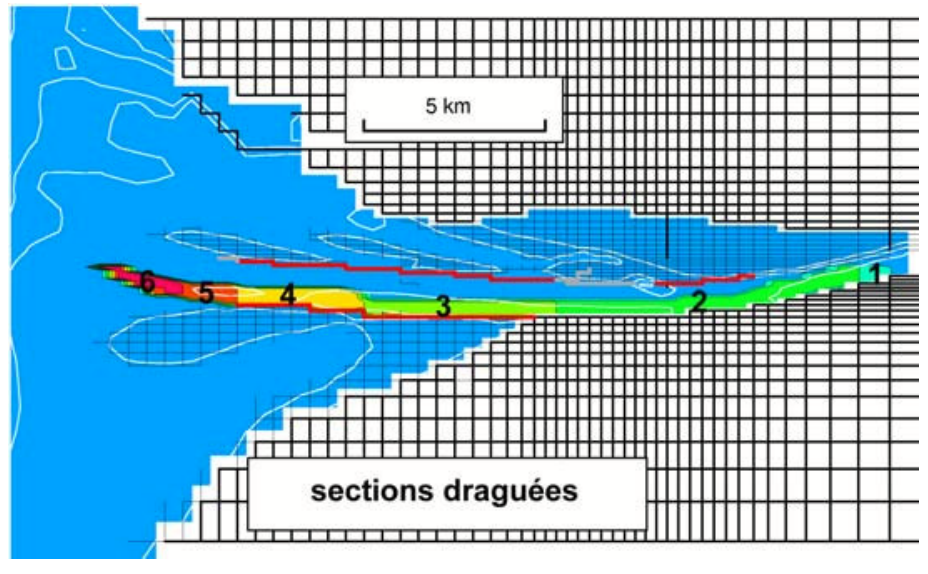

Figure 3: Définition des sections draguées à l'embouchure de la Seine dans le modèle morphodynamique SiAM-3D

A chaque pas de temps, la masse totale de sédiment dragué est distribuée en 4 mailles contiguës de la zone du Kannick (autour de l'isobathe $-3 \mathrm{~m} \mathrm{CMH}$ ), en imposant la concentration en sable et en vase dans la couche de fond de la colonne d'eau. Etant donnée sa vitesse de chute élevée, le sable se dépose rapidement sans être advecté, conformément aux observations qui montrent que la quasi-totalité $(90 \%)$ de la fraction sableuse se retrouve sur le fond après clapage (Sergent et Zhang, 2001). Par contraste, les particules de vases, qui se déposent moins rapidement, sont advectées par les courants de marée.

\section{Résultats simulés}

Le forçage hydrodynamique (marée, débit de la Seine, vent et vagues) qui est appliqué pour l'ensemble des simulations réalisées ici est le forçage de l'année 2002 (cf. Waeles, 2005), bien représentative : en particulier la variabilité saisonnière du débit de la Seine est caractéristique, avec une crue de début d'année plus importante (en intensité et en durée) que celle de fin d'année, et les vagues de hauteur significative supérieure à $1 \mathrm{~m}$ sont plus fréquentes en hiver.

Des simulations avec ou sans prise en compte des dragages sont réalisées en parallèle, l'initialisation et les conditions aux limites étant identiques. A la limite amont, les apports de sables sont considérés négligeables en raison du barrage de Poses, et la concentration de la vase, qui varie avec le débit de la Seine est issue de mesures. A la limite marine, en flot, un flux de sable à l'équilibre (avec le courant) et une concentration $\left(810^{-3} \mathrm{~g} . l^{-1}\right)$ en vase sont imposés.

Dans le but de comprendre et d'évaluer certains processus morphodynamiques, plusieurs (i.e. six) années successives (mais toujours avec le forçage hydrodynamique 2002) ont été simulées, les résultats (en termes de bathymétrie et de structure tridimensionnelle du sédiment) après une année constituant la condition initiale de l'année suivante.

\subsection{Quantités draguées simulées dans le chenal de navigation, parts de sable et de vase}

Dans un premier temps, l'analyse des bilans volumiques totaux de sédiments dragués (fig. 4) montre que les quantités simulées, variant entre 3.1 et 3.9 millions $\mathrm{de}^{3} / \mathrm{an}$, sont très proches 
des quantités effectivement draguées (fig. 2), estimés en moyenne à 3.5 millions de $\mathrm{m}^{3} / \mathrm{an}$. Sachant que le seul critère de dragage pour le modèle est le maintien de la cote du chenal de navigation, cet accord entre modèle et nature montre la capacité du modèle à simuler les bilans érosion/dépôt à moyen terme.

La représentation des volumes sédimentaires dragués par section montre une distribution satisfaisante le long du chenal. La partie la plus à l'Ouest (sections 5 et 6 ) ainsi que la partie la plus à l'Est (sections 1 et 2 , qui doivent être considérées ensemble, la section 1 n'étant constituée que de deux mailles) étant les zones nécessitant les dragages les plus importants pour maintenir une profondeur suffisante pour la navigation. Pour les tronçons 5 et 6 , les volumes dragués relativement importants lors de la première année diminuent ensuite: il s'agit probablement d'un déficit en vase, après dispersion et/ou dépôt de la crème de vase initiale. Le bilan de vase est en effet incertain, pour partie en raison des conditions aux limites mal déterminées. Par ailleurs, il faut rappeler que vase et sable ne contribuent pas de la même manière aux volumes dragués : la concentration des couches de sable dans le sédiment est d'environ $1700 \mathrm{~kg} / \mathrm{m} 3$, celle des couches de vase pouvant varier (en raison des processus de consolidation) entre une centaine et quelques centaines de $\mathrm{kg} / \mathrm{m} 3$.Enfin, il est possible que la largeur de chenal "entretenu" ait été sous-estimée à l'engainement.

Les proportions respectives de sable et de vase dans les sédiments dragués ont été évaluées (fig. 5 ; seule la $3^{\text {ème }}$ année, représentative, est montrée). Les dragages simulés sont majoritairement sableux ( $78,7 \%)$. Ce résultat est conforme aux estimations relatives aux dragages réels (70\% de sable selon Sergent et Zhang, 2001). La figure 5 fait apparaître que les dépôts sableux dans le chenal sont forcés par la marée et ont lieu majoritairement en période de vive-eau, tandis que les dépôts de vase à draguer sont liés aux forts débits de la Seine. Par exemple, on relève des dépôts de vase significatifs pour les tronçons 5 et 6 lors du pic de crue ( jour 65$)$; ces dépôts sont probablement corrélés à une position "aval" du bouchon vaseux. Les dépôts dans le chenal, qu'ils soient sableux ou vaseux, ne semblent pas sensibles aux conditions de vent et de vagues.

\subsection{Evolutions morphodynamiques tenant compte des dragages/clapages, devenir des sédiments clapés}

Lorsque les dragages et clapages sont pris en compte, un rehaussement prononcé des fonds au niveau du Kannick est logiquement obtenu (figures 7.1, 7.2 et 7.3), avec une valeur (en moyenne annuelle) comprise entre $50 \mathrm{~cm}$ et $1 \mathrm{~m}$, comparable à l'exhaussement observé à cet endroit. (en moyenne 2m-2m50 sur la période 1995-2000, Delsinne, 2005).

La représentation des différentiels bathymétriques ne permet pas de déterminer précisément le devenir des sédiments clapés au Kannick.: la tendance est un transport vers 1'Ouest/NordOuest au début, et les années suivantes un transport également vers le Sud/Sud-Est (fig. 7). Cette évolution est peut-être due à un processus morphodynamique, l'exhaussement du Kannick contribuant à ralentir les courants entre le banc d'Amfard et lui-même, et donc favorisant les dépôts dans le secteur. Ces tendances sont en accord correct avec des tendances mesurées in-situ lors de marquages radioactifs et confirmés par une modélisation locale (Sergent et Zhang, 2001). Enfin, les activités de dragage semblent avoir un impact sur l'évolution des bancs d'embouchure : les dépôts à l'Ouest des bancs seraient favorisés par les dépôts du Kannick, tendance également relevée par Delsinne (2005), en particulier concernant le banc d'Amfard au Nord du chenal de navigation. 

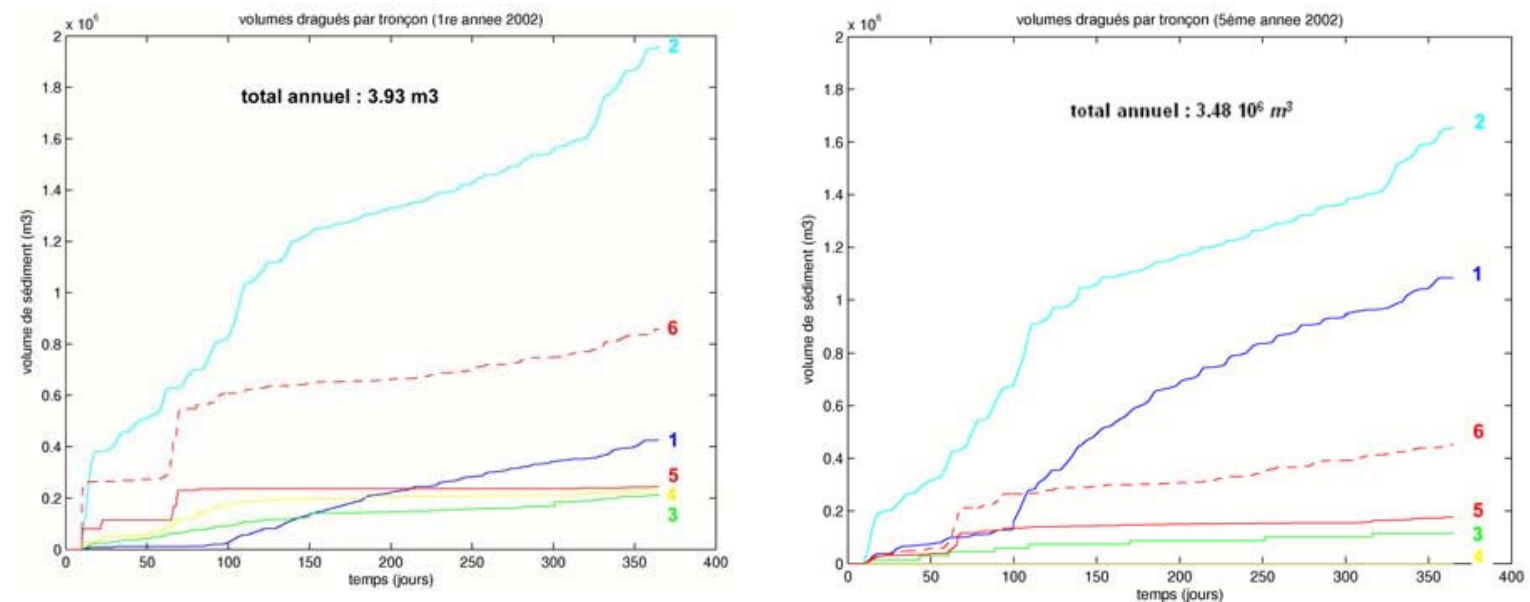

Figure 4 : Répartitions simulées des volumes de sédiment à draguer dans le chenal de navigation pour la "première" année 2002 (à gauche) et la "cinquième" année 2002 (à droite)

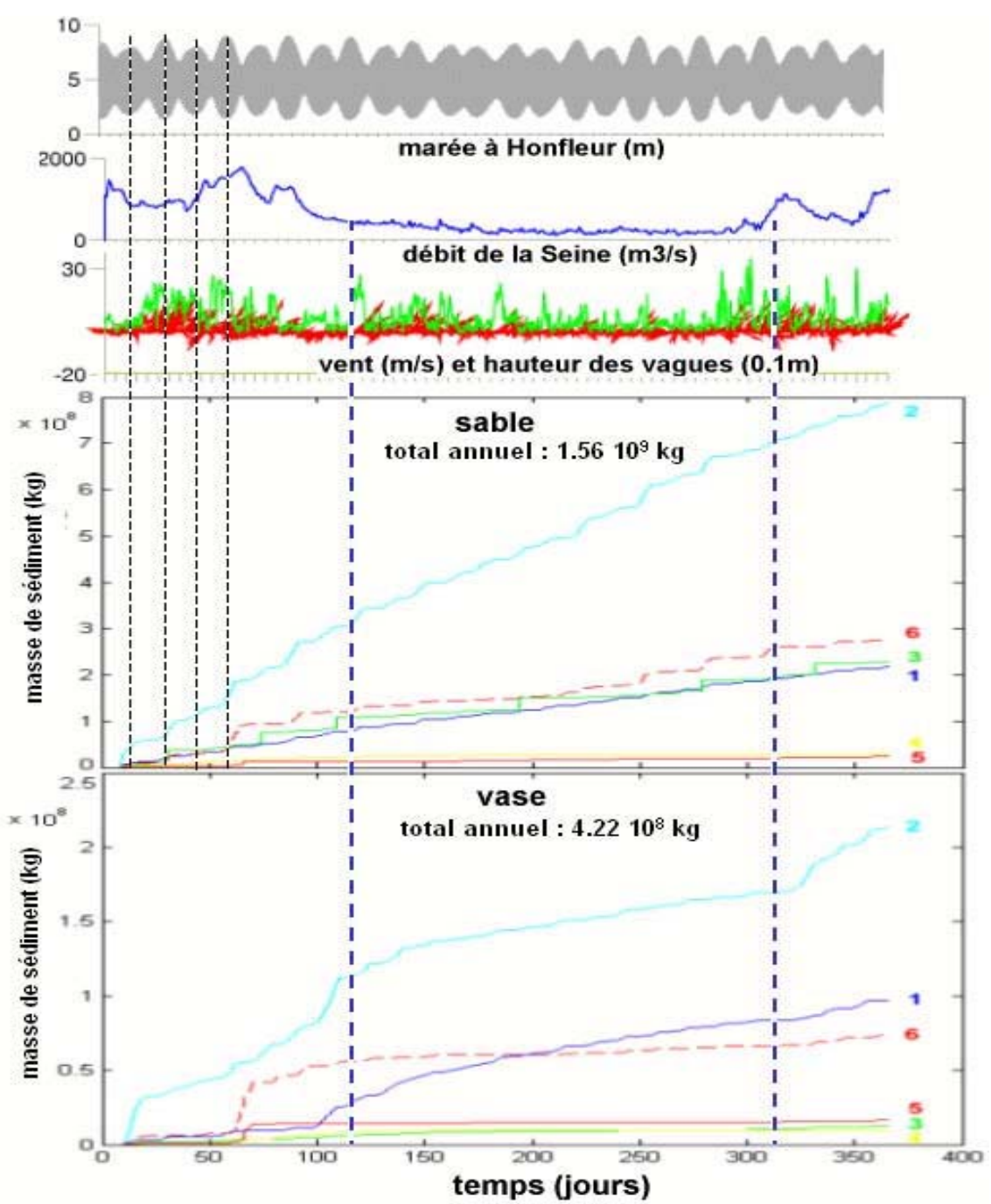

Figure 5 : haut: forçage (marée, débit de la Seine, vent et vagues) de l'année 2002 ; milieu et bas : quantités draguées cumulées de sable et de vase, durant la $3^{\text {ème }}$ année, pour les différents tronçons. Traits pointillés gris : premières viveeaux de l'année. Traits tiretés bleus : encadrent la période d'étiage. 


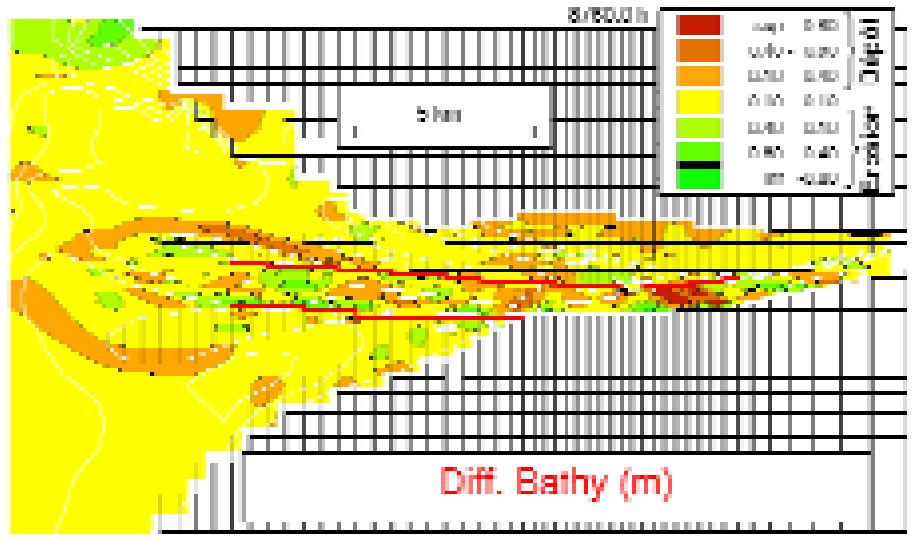

Figure 6 Exolution moephedpamleje pour un Sorgage 2002, sms dragageidapage $z^{\prime \prime}$ annde simulbs

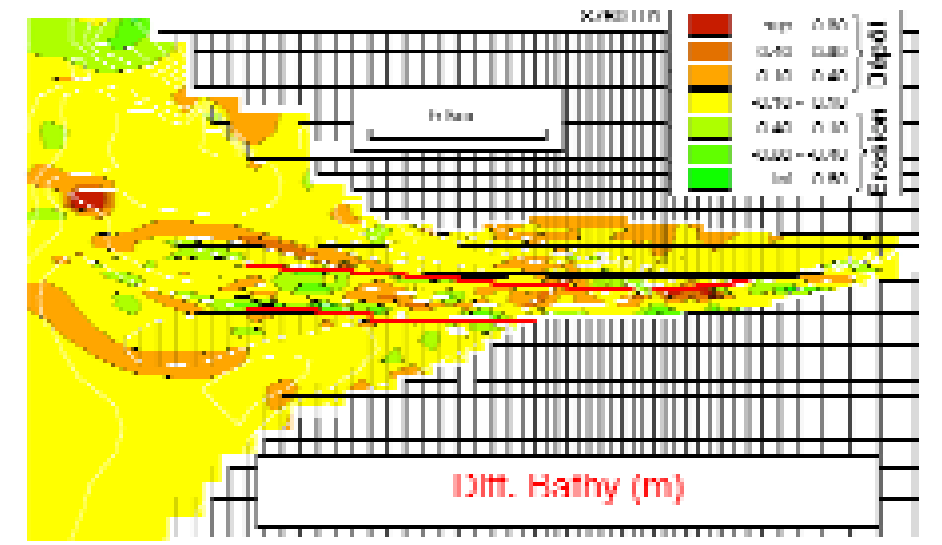

Figure 7.1 Evolubn moephodynamigoe pour un Sorgage D02 avoc dragageidapage $2^{-}$ande simulde

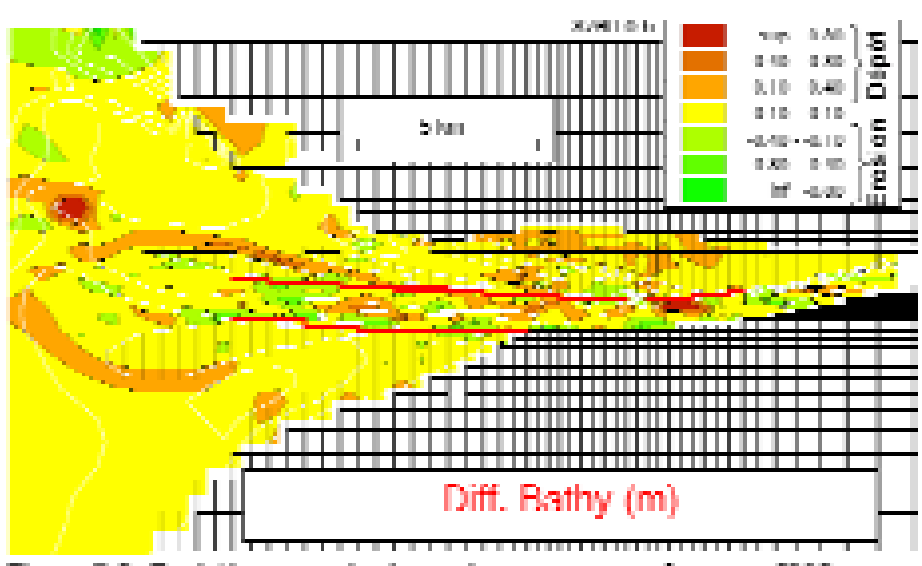

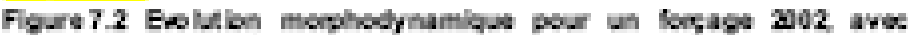
dragageidapage ga annde simulbe

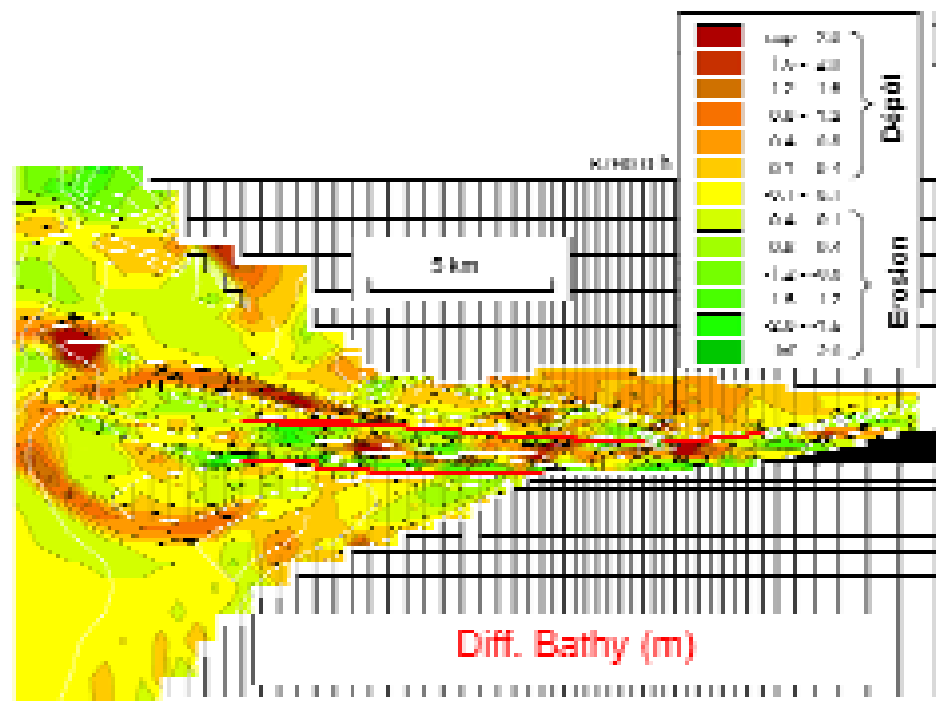

Figure 73 Evolufion morphodynamigue pour un fockage 2002, aves

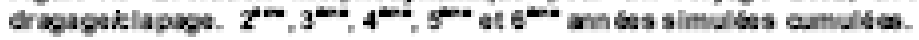

\section{$\underline{5 . \text { Conclusions }}$}

Une méthode de simulation schématique des activités de dragage et de clapage de sédiment, très proche de la démarche réelle, est proposée à l'aide du modele morphodynamique SiAM3D : le "plafond" du chenal d'accès au Port de Rouen est arasé dess lors que la cote du fond est supérieure à une cote maximale déterminée. Le clapage des sédiments ainsi dragués est réalisé en augmentant les conoentrations de sable et de vase pres du fond dans les mailles correspondant au site de rejet.

Les volumes dragués simulés (entre 3.1 et 3.9 millions de $\mathrm{m}^{3} / \mathrm{an}$ ) sont très proches des volumes réellement dragués (en moyenne de 3.5 millions de $\mathrm{m}^{3} / \mathrm{an}$, Sogreah, 1997). Conme dans la réalité, les volumes les plus importants sont dragués dans la zone amont, entre Honfleur et l'épi du Hode, et à fengainement (partie la plus à l'Ouest du chenal), bien que ces demiers soient sous-estimés. Les proportions de sable et de vase dragués (respectivement 70 $\%$ et $30 \%$ sont également conformes aux données. Les simulations d"un an avec forçages réalistes foumissent la chronologie des dragages à effectuer ainsi que leur nature et localisation: ce type de résultat pourrait servir à une gestion optimiśée des dragages (par 
exemple en cherchant à anticiper dans certains secteurs sensibles). Naturellement, l'impact d'un déplacement de la zone de clapage pourra être investigué.

La sensibilité des volumes et de la nature des sédiments dragués au forçage hydrodynamique, et en particulier aux conditions de débit de la Seine, reste à évaluer. Un marquage numérique des particules doit aussi être réalisé pour mieux appréhender le devenir des sédiments clapés au Kannick, qu'il s'agisse de sable ou de vase. Le marquage pourra également préciser la provenance des sédiments à draguer. Une discrétisation plus fine, notamment vers l'amont, permettrait enfin de mieux définir le chenal de navigation et ainsi d'évaluer plus précisément les volumes à draguer pour les différentes sections.

\section{$\underline{\text { Références : }}$}

Cugier, P. and Le Hir, P., 2002. Development of a 3D Hydrodynamic Model for Coastal Ecosystem Modelling. Application to the Plume of the Seine River (France). Estuarine, Coastal and Shelf Science, Volume 55, Issue 5, Pages 673-695.

Delsinne, N., 2005. Evolution pluri-millénaire à pluri-annuelle du prisme sédimentare de l'embouchure de la Seine. Facteurs de contrôle naturels et d'origine anthropique. Thèse de Doctorat de l'Université de Caen.

Hervouet, J.M. et Villaret, C., 2004. Simulation numérique des évolutions sédimentaires, importance d'une approche intégrée. Fascicule No. 2, VIII ${ }^{\text {ièmes }}$ Journées Nationales Génie côtier - Génie civil, Compiègne.

Le Hir, P., Ficht, A., Silva Jacinto, R.,Lesueur, P., Dupont, J.P., Lafite, R., Brenon, I., Thouvenin, B. et Cugier, P., 2001. Fine Sediment Transport and Accumulations at the Mouth of the Seine Estuary (France). Estuaries 24(6B): 950-963.

Lesourd, S., Lesueur, P., Brun-Cottan, J. C., Auffret, J. P., Poupinet, N. \& Laignel, B., 2001. Morphosedimentary evolution of a macrotidal estuary subjected to human impact ; the example of the Seine (France). Estuaries, 24 (6b), 940-949.

Migniot, C. (1989) Tassement et rhéologie des vases. La Houille Blanche, no. 1 et 2, pp. 11-29 et 95111

Sergent, P. et Zhang, B., 2001. Modélisation de l'évolution du dépôt du Kannik, Journées Scientifiques et Techniques du CETMEF, Paris.

Silva Jacinto R., 2001. Action des vagues sur les estrans et vasières. Application à l'estuaire de la Seine. Thèse de l'université de Rouen, $231 \mathrm{p}$.

Silva Jacinto R. et Burel D., 2003. Modélisation à court terme des boues de dragage rejetées par clapage. Revue Française de Génie Civil, 7/9 (2003), 20 p.

Sogreah, 1997. Port 2000. Etude sédimentologique sur modèle physique. Rapport 511471 R2.

Sogreah, 2003. Impact des clapages au Kannik sur l'évolution morphologique de l'estuaire de la Seine. Rapport 711112 R2.

Van Ledden, M., 2001. Modelling of sand-mud mixtures. Part II: A process-based sand-mud model. WL | DELFT HYDRAULICS (Z2840).

Waeles, B. et Le Hir, P, 2004. Modélisation du transport de sédiments mixtures (vase et fine sable

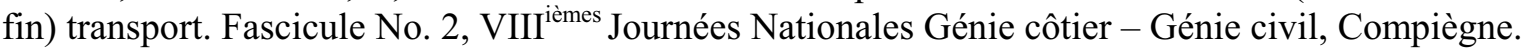

Waeles, B., 2005. Modélisation morphodynamique de l'embouchure de la Seine. Thèse de Doctorat de l'Université de Caen, 225 pages.

Waeles, B., Le Hir, P., Lesueur, P. et Delsinne, N., In press.. Modelling sand/mud transport and morphodynamics in the Seine river mouth (France): an attempt by using a process-based approach. Special issue of Hydrobiologia dedicated to the ECSA $38^{\text {th }}$ Symposium "Changes in land uses: Consequences on estuaries and coastal zones". Rouen, France, 15-17 Sept. 2004. 